\title{
Essai sur l'adaptation de la fermentation ruminale au monensin
}

\author{
L Mbanzamihigo, CJ van Nevel, DI Demeyer
}

Université de Gent, faculté des sciences agronomiques et biologiques appliquées, département des productions animales, Proefhoevestraat 10, B-9090 Melle, Belgique

(Reçu le 21 octobre 1994; accepté le 5 avril 1995)

\begin{abstract}
Résumé - L'adaptation de la fermentation ruminale au monensin a été étudiée en administrant une fois par jour $30 \mathrm{mg}$ de monensin pendant $21 \mathrm{j}$, puis $60 \mathrm{mg}$ pendant $28 \mathrm{j}$ à 2 moutons fistulés consommant chacun $300 \mathrm{~g}$ de foin et $300 \mathrm{~g}$ de concentrés 2 fois par jour à $9 \mathrm{~h}$ o0 et à $16 \mathrm{~h} 00$. Le monensin était administré dans le rumen en suspension dans l'eau juste avant le repas du matin. Notons que l'administration du monensin a été précédée d'une période pré-expérimentale et suivie d'une période post-expérimentale pendant lesquelles il n'y a pas eu d'infusion de monensin. Les paramètres ayant fait l'objet de notre étude sont : la production de méthane, le $\mathrm{pH}$, les proportions molaires des acides gras volatils (AGV), la concentration en AGV totaux, en lactate et en ammoniac dans le jus du rumen ainsi que la dégradabilité in sacco du foin. La collecte des gaz expulsés du rumen par la fistule se faisait pendant $6 \mathrm{~h}$, de $9 \mathrm{~h} 30$ ( 30 min après l'administration du monensin) à $15 \mathrm{~h}$ 30. Les autres paramètres ont été étudiés sur du jus du rumen récolté juste avant le repas du matin et l'administration du monensin, puis $2 \mathrm{~h}$ et $6 \mathrm{~h}$ après. Des incubations in vitro de $3 \mathrm{~h}$ ont été effectuées avec du contenu du rumen récolté $1 \mathrm{~h}$ après le repas et l'administration du monensin. L'analyse des résultats nous a montré que le monensin provoque aussi bien in vivo qu'in vitro une diminution de la production de méthane. Elle provoque également in vivo une augmentation de la proportion molaire du propionate, une diminution de celle de l'acétate et du butyrate, une augmentation du $\mathrm{pH}$, une diminution de la concentration en AGV totaux et en ammoniac dans le jus du rumen. Les résultats des incubations in vitro révèlent une augmentation de la production du propionate suite à l'administration du monensin ainsi qu'une diminution de la méthanogenèse. La dégradabilité in sacco de la matière sèche (MS) n'a pas changé, ceci est probablement lié au décalage horaire entre le dépôt des sachets dans le rumen et l'administration du monensin. Tant qu'on administre du monensin, les modifications citées plus haut se maintiennent, signe qu'il n'y a pas eu d'adaptation de la fermentation ruminale au monensin.
\end{abstract}

fermentation ruminale / mouton / monensin / adaptation

Summary - Adaptation of rumen fermentation to monensin administration. Adaptation of rumen fermentation to monensin feeding has been studied with rumen-fistulated sheep receiving a daily dose of $30 \mathrm{mg}$ of monensin for a period of $21 \mathrm{~d}$ followed by a $28 \mathrm{~d}$ period during which $60 \mathrm{mg}$ doses were 
administered. The ration consisted of $300 \mathrm{~g}$ of hay and $300 \mathrm{~g}$ of concentrates, fed at $9.00 \mathrm{~h}$ and 16.00 $h$. Monensin was placed in the rumen as an aqueous suspension, just prior to the morning feeding. Monensin infusion was preceded and followed by a period during which no monensin was infused. The following rumen fermentation parameters were determined: methane production, $\mathrm{pH}$, volatile fatty acids (VFA) molar proportions, total volatile fatty acid concentration, lactate and ammonia concentrations and in sacco degradability of hay. Rumen gas expelled through the fistula was collected for $6 \mathrm{~h}$ per day and analysed. Total VFA concentration, molar proportions of individual VFA, $p H$, lactate and ammonia concentration were determined on rumen contents, sampled just prior the administration of monensin and 2 and $6 \mathrm{~h}$ later. In vitro incubations of $3 \mathrm{~h}$ were carried out with rumen fluid, sampled $1 \mathrm{~h}$ after feeding. In vivo and in vitro methane production was decreased by monensin feeding. The molar proportion of propionate in the rumen was increased, while acetate and butyrate percentages were lowered. The total VFA and ammonia concentrations were also decreased by monensin, but $p H$ values were increased. In vitro production of propionate was stimulated by monensin administration and methanogenesis decreased. The organic matter in sacco degradability was not affected, probably because of the time difference between the introduction of bags and monensin in the rumen. These modifications of rumen fermentation persisted as long as monensin was given, indicating that in this experiment, there was no adaptation to the ionophore.

rumen fermentation / sheep / monensin / adaptation

\section{INTRODUCTION}

De nombreux travaux ont été effectués sur I'utilisation des antibiotiques pour orienter la fermentation dans le rumen. Parmi ces antibiotiques, le monensin, isolé à partir d'une culture de Streptomyces cinnamonensis (Haney et Hoehn, 1967) est le seul ionophore légalement incorporé dans les aliments des ruminants en Belgique.

L'amélioration des paramètres zootechniques due au monensin, rapportée par de nombreux auteurs, est liée à une diminution de la méthanogenèse (Joyner et al, 1979 ; Thornton et Owens, 1981 ; Crutzen et al, 1986) et à une augmentation de la proportion molaire du propionate associée à une diminution de la proportion molaire de l'acétate et parfois du butyrate (Raun et al, 1976 ; Richardson et al, 1976 ; Chalupa et al, 1980 ; Dyer et al, 1980 ; Horn et al, 1981 ; Stanier et Davies, 1981). Cette amélioration est également consécutive à une baisse de la dégradation des protéines alimentaires au niveau du rumen (Poos et al, 1979 ; Sakauchi et al, 1979 ; Horn et al, 1981).
Des études réalisées sur une longue durée ont prouvé que les modifications des proportions molaires des différents acides gras volatils (AGV) provoquées par le monensin se maintiennent tant qu'on administre encore ce produit (Raun et al, 1976 ; Richardson et al, 1976 ; Dyer et al, 1980 ; Horn et al, 1981). D'autres auteurs dont Rumpler et al (1986), Carmean et Johnson (1990) rapportent une reprise de la production de méthane respectivement au $12^{\mathrm{e}}$ et au $9^{\mathrm{e}}$ jour de l'administration du monensin, signe d'une adaptation à l'ionophore. En tenant compte de la stœchiométrie de la fermentation dans le rumen où une augmentation du propionate s'accompagne nécessairement d'une diminution de la production de méthane (Wolin, 1960), il existe une contradiction quant à l'adaptation au monensin même si dans le cas qui nous concerne les paramètres cidessus cités (méthane et AGV) n'ont jamais été étudiés simultanément. Ainsi donc, contrairement aux auteurs cités plus haut, nous nous sommes proposé d'étudier l'adaptation au monensin en mesurant concomitamment certains paramètres de la fermentation ruminale sur une période relativement longue, aussi bien in vivo qu'in vitro. 


\section{MATÉRIEL ET MÉTHODES}

\section{Schéma expérimental}

L'adaptation de la fermentation ruminale au monensin a été étudiée en administrant une fois par jour $30 \mathrm{mg}$ de monensin pendant $21 \mathrm{j}(\mathrm{P} 30)$, puis $60 \mathrm{mg}$ pendant 28 j $(P 60)$ à 3 moutons fistulés dont 1 est mort et n'a pas été remplacé. Les paramètres ayant fait l'objet de notre étude sont : la production de méthane, le $\mathrm{pH}$, les proportions molaires des acides gras volatils (AGV), la concentration en AGV totaux, en lactate et en ammoniac dans le jus du rumen ainsi que la dégradabilité in sacco du foin. L'administration du monensin avait été précédée d'une période témoin de $21 \mathrm{j}(\mathrm{PT})$ et suivie d'une période postexpérimentale de $31 \mathrm{j}(\mathrm{PA})$ pendant lesquelles il n'y a pas eu d'administration du monensin. Notons que des incubations in vitro de $3 \mathrm{~h}$ ont été effec- tuées au cours des 3 périodes avec du contenu du rumen récolté $1 \mathrm{~h}$ après le repas et l'administration du monensin (tableau I).

\section{Régime et animaux}

Trois béliers castrés de race Texel mixte A, B et $C$, porteurs d'une fistule permanente au niveau du rumen, âgés respectivement de 45,45 et 39 mois, pesant 98,5, 105,5 et $89 \mathrm{~kg}$ respectivement, ont été utilisés. Ces animaux ont été maintenus dans des cages métaboliques où ils recevaient $300 \mathrm{~g}$ de foin et $300 \mathrm{~g}$ de concentrés (voir la composition dans le tableau II) 2 fois par jour et par animal à $9 \mathrm{~h}$ et à $16 \mathrm{~h}$.

Une dose de $30 \mathrm{mg}$ de monensin (Monensin sodium salt $90-95 \%$ SIGMA, Belgique) en suspension dans $30 \mathrm{ml}$ d'eau du robinet était administrée à chaque animal par la fistule une fois par

Tableau I. Plan expérimental.

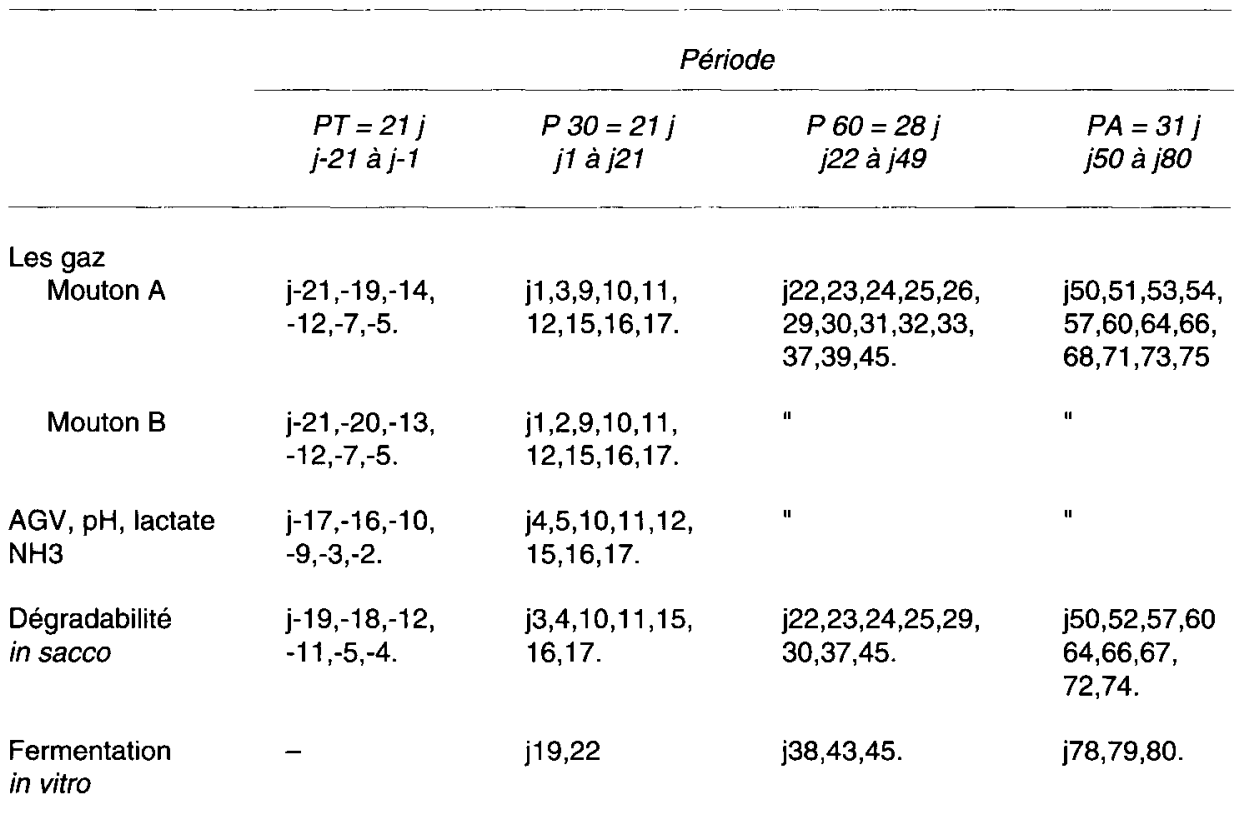

$\mathrm{j}-x$ ou $\mathrm{j} x$ : $x \mathrm{j}$ avant ou après la fin de la période témoin ; PT : période témoin ; $\mathrm{P} 30$ : période d'administration de $30 \mathrm{mg}$ de monensin ; $\mathrm{P} 60$ : période d'administration de $60 \mathrm{mg}$ de monensin ; PA : période après l'administration du monensin. 
Tableau II. Composition du régime.

\section{Ingrédients}

Foin $\quad$ Foin de prairie de qualité moyenne

Concentré Avoine $=37 \%$, maïs $=32 \%$, mélasse $=2 \%$, tourteau de soja $=14,5 \%$, luzerne $=5 \%$, graisse animale $=1 \%$, phosphate bicalcique $=2 \%$, craie $=5 \%, \mathrm{NaCl}=1 \%$, complexe vitamines-minéraux $=0,5 \%$

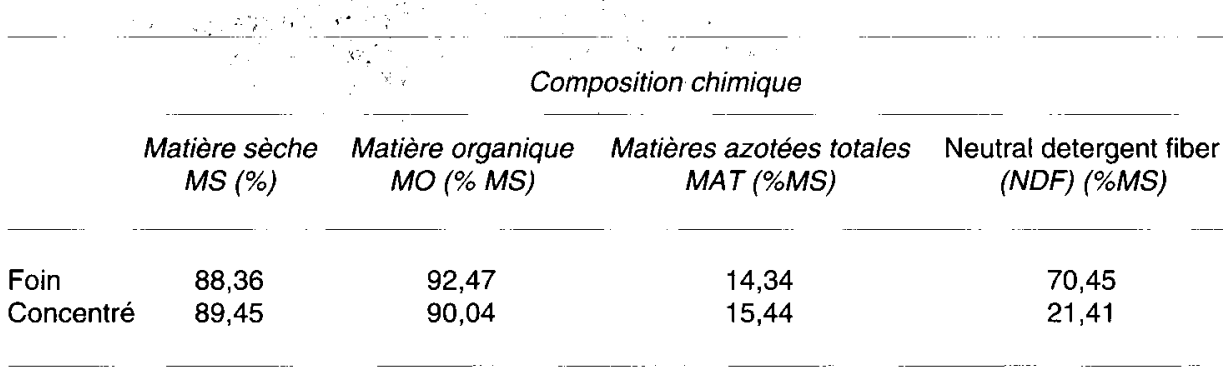

jour à $9 \mathrm{~h}$ (juste avant le repas du matin). La dose de $30 \mathrm{mg}$ de monensin n'ayant pas provoqué une diminution suffisante de la production de méthane, nous avons décidé d'en administrer $60 \mathrm{mg}$. Au cours de la période d'administration de $60 \mathrm{mg}$ de monensin, le mouton $\mathrm{C}$ a refusé d'ingérer sa ration quotidienne pour une raison inconnue. Quelques jours plus tard, l'animal est mort. L'intoxication par le monensin est possible mais pas très probable car, la $\mathrm{DL}_{50}$ de cet ionophore, administrée en une fois à des moutons est de 11,9 $\mathrm{mg} / \mathrm{kg}$ de poids vif (Todd et al, 1984), dépassant de loin 10 fois la dose administrée.

\section{Prélèvements et mesures}

\section{Les gaz}

Après une période de mise en régime de 30 j, des gaz expulsés par la fistule ont été collectés aux jours indiqués dans le tableau I, de $9 \mathrm{~h} 30$ à $15 \mathrm{~h} 30(6 \mathrm{~h})$. La collecte se faisait à l'aide du dispositif décrit par Van Nevel et al (1970), modifié pour l'adapter à 3 moutons et pour recueillir ainsi un volume plus important de gaz en utilisant pour chaque mouton une grande colonne en PVC et une petite en verre pouvant contenir respectivement $17,5 \mathrm{I}$ et $7,5 \mathrm{I}$. La quantité de méthane dans le gaz total était déterminée à l'aide d'un chromatographe relié au dispositif (Van Nevel et al, 1970).

La méthode utilisée pour collecter les gaz expulsés par la fistule nous a permis de récolter non pas la totalité, mais une grande proportion du gaz total produit au niveau du rumen de chaque mouton au cours de la période considérée. En effet, une partie des gaz de fermentation a été évacuée par éructation et par respiration (Van Nevel et al, 1970). À titre indicatif, un calcul stoechiométrique à partir de la matière organique ingérée (Demeyer, 1991) permet d'estimer la production journalière $(24 \mathrm{~h}$ ) à environ 44 I de méthane soit 11 I en $6 \mathrm{~h}$. D'après une relation établie par Shibata et al (1992) entre la production de méthane et la quantité de matière sèche (MS) ingérée par un ruminant qui reçoit $150 \%$ de ses besoins d'entretien, le repas du matin est susceptible de produire 11,83 I de méthane. Nous pouvons donc estimer que les 8,21 I que nous avons collectés en moyenne par mouton en $6 \mathrm{~h}$, au cours de la période témoin, représentent environ $70 \%$ de la production attendue.

\section{Les AGV, le lactate, l'ammoniac et le pH}

Le système de collecte des gaz nous permettait en même temps de prendre des échantillons de jus du rumen à $9 \mathrm{~h}$ (avant l'administration du monensin ou $24 \mathrm{~h}$ après), à $11 \mathrm{~h}$ ( $2 \mathrm{~h}$ après l'ad- 
ministration du monensin), et à $15 \mathrm{~h}$ ( $6 \mathrm{~h}$ après l'administration du monensin) pour mesurer les paramètres cités plus haut. Les acides gras volatils (AGV), les concentrations en ammoniac et en acide lactique ont été déterminés comme décrit par Van Nevel et Demeyer (1977a, b) et Demeyer et Van Nevel (1979), le pH a été déterminé à l'aide d'un pH-mètre standard PHM62 (Radiometer, Copenhagen).

\section{La dégradabilité in sacco}

Des sachets en polyester (Solana, Edegem, Belgique), ayant des pores de $50 \mu$ et $36 \%$ de surface libre ont été utilisés. Trois sachets contenant $2 \mathrm{~g}$ de foin (le même foin que celui de la ration) ont été introduits dans le rumen de chaque mouton à $15 \mathrm{~h}$ ( $6 \mathrm{~h}$ après l'administration du monensin) aux jours indiqués dans le tableau I pour être retirés $24 \mathrm{~h}$ après. Le foin utilisé était préalablement moulu avec un moulin Brabender et tamisé pour ne garder que les particules ayant des dimensions de $212 \mu$ à $1 \mathrm{~mm}$. Après le retrait des sachets du rumen, ils étaient rincés à la machine à laver pendant 20 min puis séchés à $65^{\circ} \mathrm{C}$ pendant $48 \mathrm{~h}$ pour en déduire finalement le pourcentage de matière sèche (MS) dégradée dans le rumen.

\section{Fermentation in vitro}

Au cours des périodes d'administration de $30 \mathrm{mg}$ de monensin ( $P$ 30), de $60 \mathrm{mg}$ de monensin ( $P$ 60) et après l'arrêt de l'administration de l'ionophore (PA), aux jours indiqués dans le tableau I, $100 \mathrm{ml}$ du contenu du rumen de chaque mouton étaient récoltés $1 \mathrm{~h}$ après l'administration du monensin et filtrés grossièrement à l'aide d'une grille métallique dont les mailles mesurent $1 \mathrm{~mm}$ de diamètre. Au total $25 \mathrm{ml}$ du filtrat en P 30 et PA d'une part, $10 \mathrm{ml}$ en $\mathrm{P} 60$ et PA d'autre part ont été incubés pendant $3 \mathrm{~h}$ avec du $\mathrm{CO}_{2}$ à $100 \%$ après avoir été préalablement mélangés respectivement avec 25 $\mathrm{ml}$ et $40 \mathrm{ml}$ de Burroughs $10 \%(\mathrm{pH}=6,8)$ (Burroughs et $\mathrm{al}, 1950$ ). L'incubation a été faite à $39^{\circ} \mathrm{C}$ dans un bain-marie faisant des mouvements réguliers de $3 \mathrm{~cm}$. Les produits de fermentation (AGV, méthane) ont été déterminés et le bilan d'hydrogène métabolique a été calculé à l'aide des formules de Demeyer (1991).

Après avoir fait des incubations simultanées de $25 \mathrm{ml}$ et de $10 \mathrm{ml}$ du contenu du rumen à la fin de la période qui suit l'arrêt de l'administration du monensin (PA), nous avons constaté que les résultats obtenus avec $25 \mathrm{ml}$ ne diffèrent de ceux obtenus avec $10 \mathrm{ml}$ que par le facteur de dilution $(2,5)$.

\section{Traitement des résultats}

Chaque jour de la période expérimentale étant susceptible d'être différent des autres quant aux paramètres mesurés, nous avons comparé chaque jour de cette période avec la moyenne de la période témoin plus ou moins le double de l'écart type. Les valeurs situées hors de cet intervalle sont considérées comme significativement différentes de celles de la période témoin. Le monensin ayant provoqué des effets similaires à $9 \mathrm{~h}, 11 \mathrm{~h}$ et $15 \mathrm{~h}$, avec une intensité variable selon les moutons, seuls les résultats de $11 \mathrm{~h}$ seront présentés mouton par mouton.

\section{RÉSULTATS}

\section{Les gaz}

La production des gaz est présentée sur la figure 1. L'administration de $30 \mathrm{mg}$ de monensin ne provoque une diminution significative de la production de méthane que chez le mouton $B$, alors que $60 \mathrm{mg}$ de monensin entraînent une baisse significative de la production de méthane chez les 2 moutons. Cette baisse se maintient tant que l'administration du monensin se poursuit. L'arrêt de l'administration de ce produit est suivi d'une chute puis d'un recouvrement progressif de la production de méthane qui atteint le niveau de la période témoin (PT) après $4 \mathrm{j}$ pour le mouton $A$ et $16 \mathrm{j}$ pour le mouton $B$. La production du gaz total évolue dans le même sens que celle du méthane. Notons que la variabilité de la production de méthane pendant la période témoin (PT) est plus importante pour le mouton $A$ que pour le mouton $B$, ce qui influe sur le seuil de signification et par conséquent sur le nombre de valeurs significatives. 

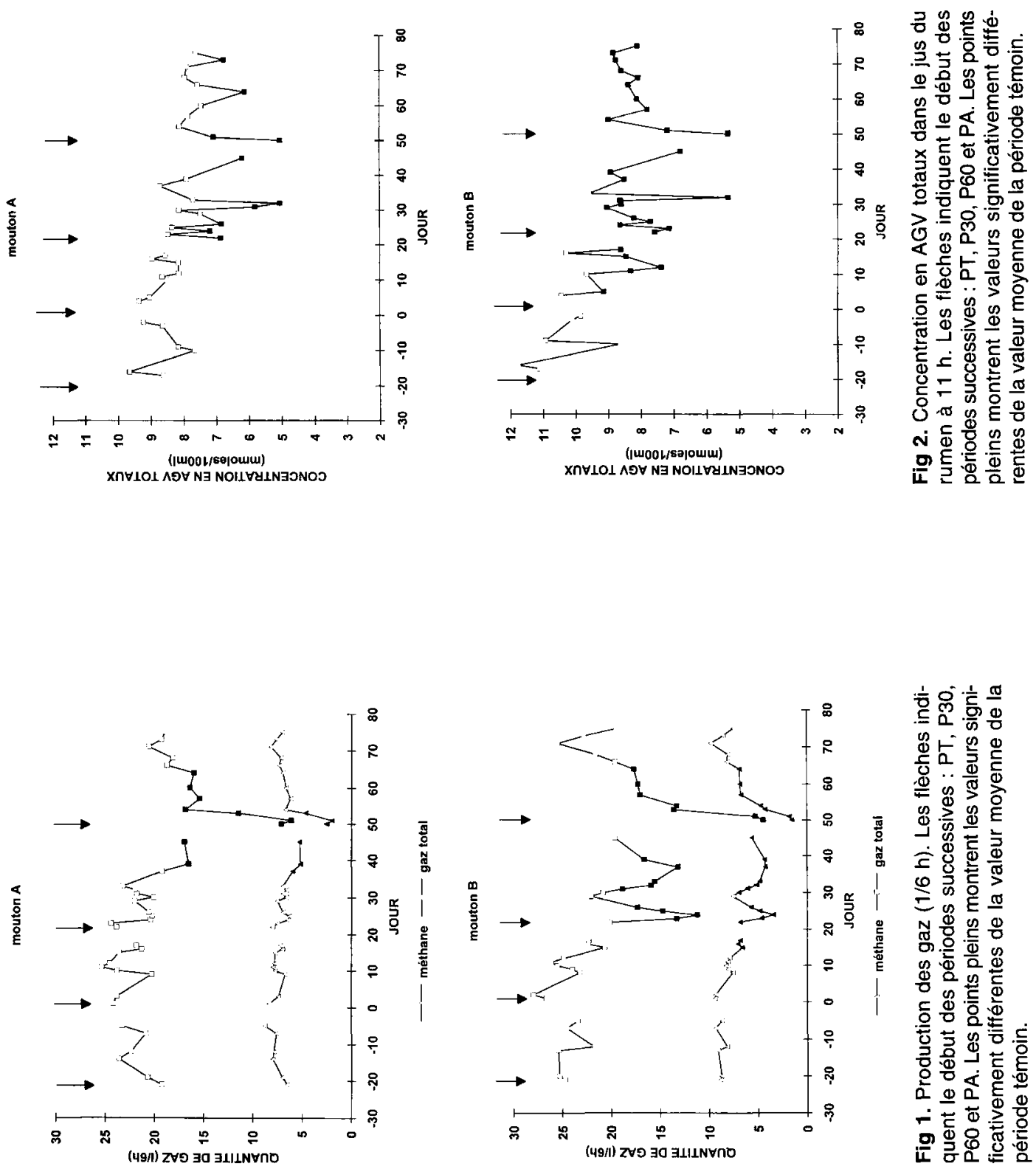


\section{Les AGV, le pH, le lactate}

La figure 2 montre que le monensin entraîne une diminution de la concentration en AGV totaux dans le jus du rumen des 2 moutons même si elle n'est pas significative en période $P 30$ chez le mouton A. Les proportions molaires des différents AGV dans le rumen présentées sur la figure 3 montrent que le monensin provoque une augmentation de la proportion molaire du propionate, une diminution de la proportion molaire de l'acétate et du butyrate. Comme pour la production de méthane et la concentration en AGV totaux, ces modifications intervenues dans les proportions molaires des différents AGV ne sont pas toujours significatives. Les modifications de la fermentation ruminale citées plus haut se maintiennent pendant toute la durée du traitement, mais l'arrêt de l'administration du monensin entraîne une évolution brusque vers les valeurs de la période témoin.

L'administration du monensin ne provoque une augmentation du $\mathrm{pH}$ du contenu du rumen qu'en période d'administration de $60 \mathrm{mg}$ de monensin. Les valeurs moyennes $\mathrm{du} \mathrm{pH}$ à $11 \mathrm{~h}$ passent de 6,43 en période PA à 6,57 en période $P 60$ chez le mouton $A$ et de 6,30 à 6,44 pour les mêmes périodes chez le mouton $B$.

La concentration en acide lactique du jus de rumen récolté $2 h, 6$ h et 24 h après l'administration du monensin reste négligeable (inférieure à $1 \mu \mathrm{mole} / \mathrm{ml}$ ).

\section{L'ammoniac}

Comme le montre la figure 4, la concentration en ammoniac dans le jus du rumen récolté $2 \mathrm{~h}$ après l'administration du monensin diminue par rapport à la période témoin. Cette diminution qui est significative chez tous animaux à la dose de $60 \mathrm{mg}$ de monensin ne l'est que chez le mouton $B$ en période
P 30. La baisse de la concentration en ammoniac est maintenue tant que l'administration de cet antibiotique se poursuit. L'arrêt de l'administration du monensin entraîne une augmentation progressive de la concentration en ammoniac dans le jus du rumen.

\section{La dégradabilité in sacco}

Le tableau III montre que l'administration du monensin ne provoque pas de changements importants de la dégradabilité in sacco de la matière sèche (MS) dans le rumen, les sachets ayant été déposés $6 \mathrm{~h}$ après l'administration du monensin. Néanmoins, nous constatons qu'après l'arrêt de l'administration de ce produit cette dégradabilité chute d'abord et augmente par la suite.

\section{Fermentations in vitro}

Le tableau IV rapporte la production des $A G V$, la production de méthane ainsi que le bilan d'hydrogène métabolique après incubation du contenu du rumen des moutons $A$ et $B$.

En comparant la période PA avec les périodes $P 30$ et $P 60$, nous constatons que le monensin entraîne une diminution modérée de la production de méthane, une augmentation de la production du propionate alors que la production des autres AGV est variable, et ce tant que le monensin continue à être administré. Notons que l'augmentation de la production du propionate induite par le monensin est plus importante pour le mouton $B$ que pour le mouton $A$ au cours de l'administration de $30 \mathrm{mg}$ de monensin. Les bilans d'hydrogène métabolique sont normaux ; cela indique que les incubations sont conformes au modèle de fermentation proposé par Demeyer (1991) et qu'il n'y a donc 

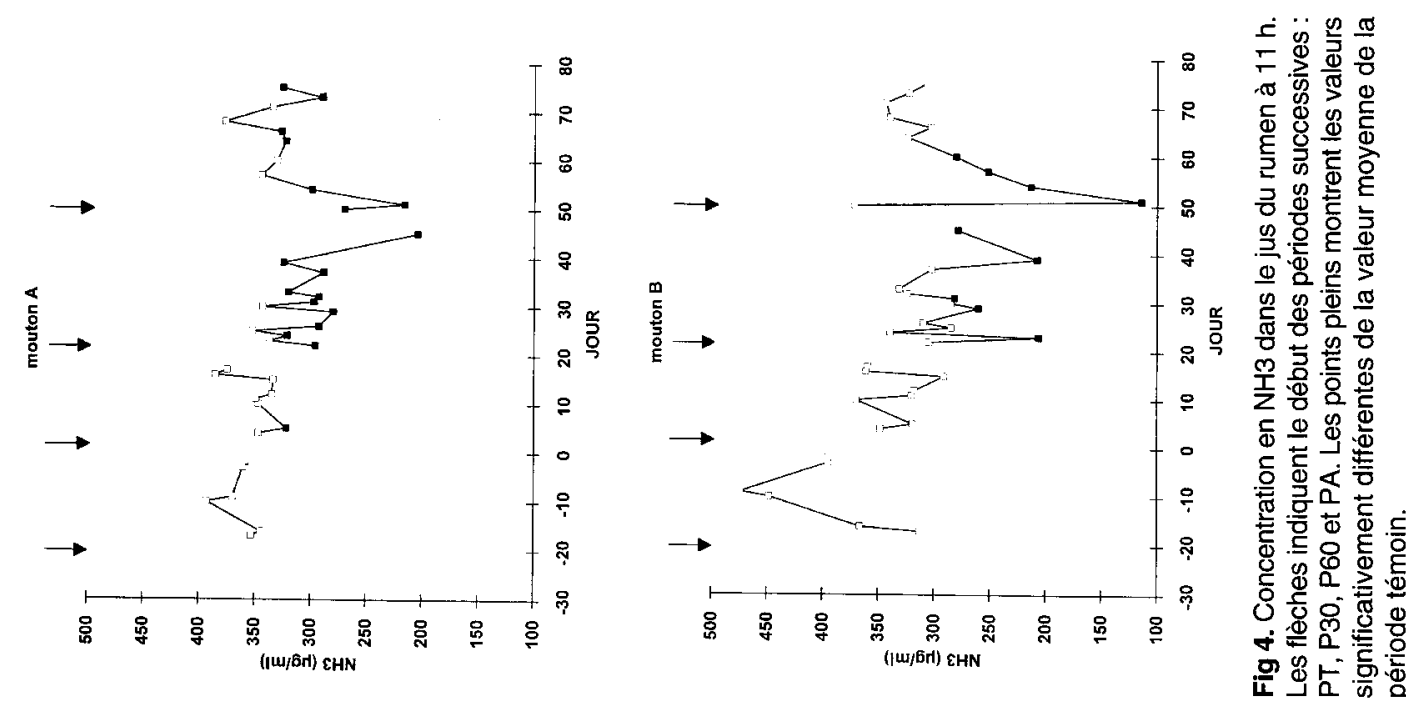

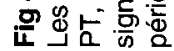

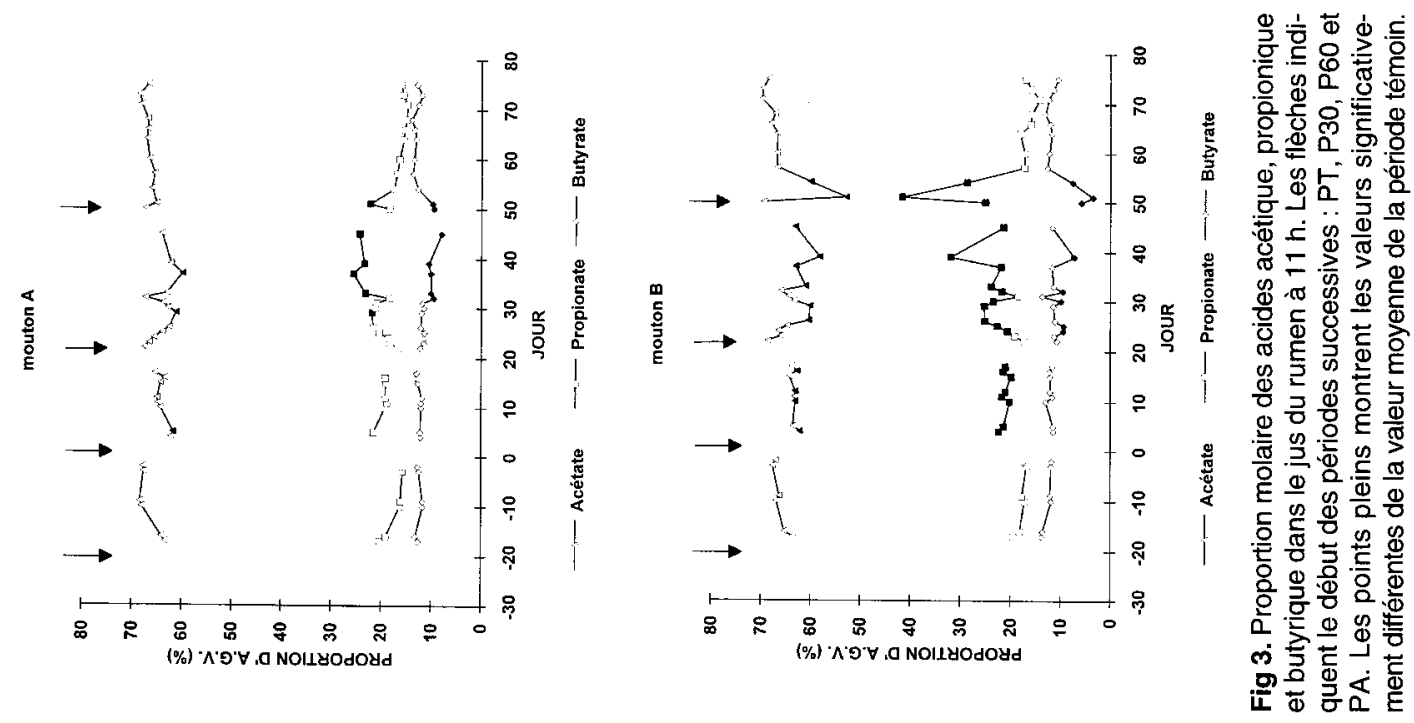


Tableau III. Dégradabilité au temps $24 \mathrm{~h}$ de la matière sèche (\% MS) mesurée in sacco.

\begin{tabular}{|c|c|c|c|}
\hline \multirow[t]{2}{*}{ Période } & \multirow[t]{2}{*}{ Jour } & \multicolumn{2}{|c|}{ Dégradabilité (\%) } \\
\hline & & Mouton A & Mouton B \\
\hline \multirow[t]{6}{*}{ PT } & $\mathrm{j}-19$ & 49,6 & - \\
\hline & $\mathrm{j}-18$ & 43,9 & 36,2 \\
\hline & $\mathrm{j}-12$ & - & 38,8 \\
\hline & $\mathrm{j}-11$ & 46,2 & 43,8 \\
\hline & $\mathrm{j}-5$ & 44,8 & 40,9 \\
\hline & $\mathrm{j}-4$ & - & 40,9 \\
\hline \multicolumn{2}{|c|}{ moyenne (et) } & $46,1(2,5)$ & $40,1(2,8)$ \\
\hline \multirow[t]{7}{*}{ P 30} & j3 & 42,5 & 45,7 \\
\hline & j4 & 42,5 & 40,6 \\
\hline & j10 & 44,3 & 40,9 \\
\hline & j11 & - & 38,8 \\
\hline & j15 & - & 39,4 \\
\hline & j16 & - & 43 \\
\hline & $j 17$ & 45,1 & 41,1 \\
\hline \multicolumn{2}{|c|}{ moyenne (et) } & $43,6(1,3)$ & $41,4(2,3)$ \\
\hline \multirow[t]{7}{*}{$P 60$} & j22 & - & 43,9 \\
\hline & j23 & 41,7 & - \\
\hline & $\mathrm{j} 24$ & 46,3 & 46,1 \\
\hline & j25 & - & 48 \\
\hline & $\mathrm{j} 29$ & 41,3 & - \\
\hline & $\mathrm{j} 37$ & 50,6 & 50,2 \\
\hline & $\mathrm{j} 45$ & 48,7 & - \\
\hline \multicolumn{2}{|c|}{ moyenne (et) } & $45,7(4,1)$ & $47,1(2,7)$ \\
\hline \multirow[t]{9}{*}{ PA } & j50 & 35,2 & 26,8 \\
\hline & j52 & 46 & 45,5 \\
\hline & j57 & 50 & 45,5 \\
\hline & $j 60$ & 50,1 & 45,6 \\
\hline & j64 & 53,2 & 49,7 \\
\hline & j66 & 50,4 & 49,4 \\
\hline & j67 & 47,4 & 44,9 \\
\hline & j72 & 52,8 & 48,2 \\
\hline & j74 & - & 45,9 \\
\hline \multicolumn{2}{|c|}{ moyenne (et) } & $48,1(5,8)$ & $44,6(6,9)$ \\
\hline
\end{tabular}

MS : matière sèche ; et : écart type ; j-x; jx; $P T$; $P$ 30 ; P 60 ; PA : voir tableau I. aucun produit de fermentation réduit qui n'aurait pas été analysé.

\section{DISCUSSION ET CONCLUSION}

L'administration du monensin provoque une diminution de la production de méthane aussi bien in vivo qu'in vitro bien qu'elle ne soit pas toujours significative. En effet, cette diminution est significative en période $P 60$ chez tous les moutons alors qu'elle ne l'est pas en période $P 30$ chez le mouton $A$, suite, non seulement à la différence de sensibilité au monensin entre les 2 moutons, mais aussi à une grande variabilité de la production de méthane par ce mouton au cours de la période témoin. La diminution de la méthanogenèse provoquée par le monensin aussi bien in vivo qu'in vitro avait été rapportée par de nombreux auteurs dont Van Nevel et Demeyer (1977a), Jouany et Sénaud (1978), Joyner et al (1979), Thornton et Owens (1981) et Crutzen et al (1986).

Tant qu'on administre du monensin, la quantité de méthane produite reste diminuée par rapport à celle de la période témoin contrairement aux résultats de Rumpler et al (1986) et Carmean et Johnson (1990) qui rapportent une reprise totale de la production de méthane respectivement au 12 et au $9^{\mathrm{e}}$ jour de l'administration du monensin. Rappelons que Rumpler et al (1986) ont travaillé avec des bœufs consommant 4066 $\mathrm{g}$ de matière sèche (MS) dont $70 \%$ de grains de céréales concassés, et recevant $55,6 \mathrm{mg}$ de monensin $/ \mathrm{kg}$ d'aliment et que Carmean et Johnson (1990) ont utilisé des bœufs recevant une ration constituée de $90 \%$ de concentrés, et une dose de $26 \mathrm{mg}$ de monensin/kg d'aliment.

L'arrêt de l'administration du monensin est suivi d'un recouvrement progressif de la méthanogenèse qui atteint le niveau de la période témoin après $4 \mathrm{j}$ (mouton $\mathrm{A}$ ) ou 16 j (mouton B). Cette différence dans la période de recouvrement de la méthano- 
Tableau IV. Incubations in vitro: production des acides gras volatils (AGV), du méthane et bilan d'hydrogène métabolique (moutons $\mathrm{A}$ et $\mathrm{B}$ ).

\begin{tabular}{|c|c|c|c|c|c|c|}
\hline $\begin{array}{l}\text { Période } \\
\qquad \text { Jours }\end{array}$ & $A^{a}$ & $p a$ & $B^{a}$ & $A G V$ tot $^{b}$ & $\mathrm{CH}_{4}{ }^{\mathrm{c}}$ & Bilan $2 H^{d}$ \\
\hline
\end{tabular}

\section{Mouton A}

$\begin{array}{lllllllr}\text { P30 } & \text { j19e } & 1152 & 214 & 317 & 1717 & 514 & 82,3 \\ & \mathrm{j} 22 & 1106 & 225 & 334 & 1088 & 742 & 108,3\end{array}$

Moyenne (et) $1129,0(32,5) \quad 219,5(7,8) \quad 325,5(12) \quad 1402,5(444,8) 628(161,2) \quad 95,3(18,4)$

\begin{tabular}{|c|c|c|c|c|c|c|}
\hline$P 60 \quad j 38$ & 1079 & 360 & 280 & 1050 & 518 & 92,1 \\
\hline $\mathrm{j} 43$ & 1177 & 469 & 176 & 1277,5 & 531 & 96 \\
\hline j45 & 1142 & 572 & 143 & 1528,8 & 544 & 105 \\
\hline
\end{tabular}

Moyenne (et) $1132,7(49,7) \quad 467,0(106) \quad 199,7(71,5) 1285,4(239,5) 531(13) \quad 98,0(6,6)$

$\begin{array}{llllllll}\text { PA } & \text { j78 } & 1109 & 240 & 325 & 1252 & 697 & 104,2 \\ & \text { j79 } & 1176 & 198 & 313 & 1304 & 719 & 102,5 \\ & \text { j80 } & 1161 & 177 & 331 & 1398 & 726 & 103,2\end{array}$

Moyenne (et) $1148,7(35,2) \quad 205,0(32,1) \quad 323,0(9,2) \quad 1318,0(74) \quad 714(15,1) \quad 103,3(0,9)$

\section{Mouton B}

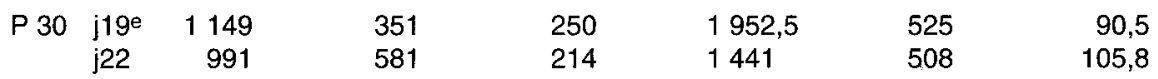

Moyenne (et) $1070,0(111,7) 466,0(162,6) 232(25,5) \quad 1696,7(361,7) \quad 516,5(12) \quad 98,2(10,9)$

\begin{tabular}{|c|c|c|c|c|c|c|}
\hline$P 60 \quad j 38$ & 1064 & 582 & 177 & 1775 & 342 & 84 \\
\hline j43 & 1148 & 350 & 251 & 1776 & 633 & 102 \\
\hline$j 45$ & 1136 & 322 & 271 & 1105 & 578 & \\
\hline
\end{tabular}

Moyenne (et) $1116,0(45,4) \quad 418,0(142,7) 233(49,5) \quad 1552,0(387,1) \quad 517,7(154,6) \quad 93,9(9)$

$\begin{array}{llllllll}\text { PA } & \text { j78 } & 1233 & 216 & 276 & 1348 & 750 & 105,2 \\ & j 79 & 1192 & 242 & 283 & 1176 & 721 & 104,8 \\ & \text { j80 } & 1146 & 273 & 290 & 1826 & 656 & 100,6\end{array}$

Moyenne (et) $1190,3(43,5) \quad 243,7(28,5) \quad 283(7) \quad 1450,0(336,8) \quad 709,0(48,1) \quad 103,5(2,5)$

a $A, P, B$ : acides acétique, propionique et butyrique en $\mu$ moles par mmoles d'hexose fermenté $\left(C_{6} f\right)\left(C_{6} f=\right.$ $\mathrm{A} / 2+\mathrm{P} / 2+\mathrm{B}$ ) (Demeyer, 1991) ; ${ }^{b}$ acides gras volatils totaux en $\mu$ moles par incubation ; $\mathrm{c}$ production de méthane en $\mu$ moles par $100 \mu$ moles de $C_{6} f$; d bilan d'hydrogène métabolique (\%); e jx (voir tableau I). 
genèse après l'arrêt de l'administration du monensin est due à la variabilité de la production journalière de méthane au cours de la période témoin ( $\mathrm{PT}$ ) mais aussi à une sensibilité différente des 2 moutons à l'ionophore.

Le monensin administré in vivo entraîne une diminution de la concentration en AGV totaux, une augmentation de la proportion molaire du propionate, une baisse de celle de l'acétate et du butyrate. Ces changements qui ne sont pas toujours significatifs, se maintiennent tant que l'administration de l'ionophore se poursuit ; ceci est en accord avec les résultats d'autres auteurs dont Richardson et al (1976), Beede et Farlin (1977), Chalupa et al (1980), Horn et al (1981), Stanier et Davies (1981). Nous avons également constaté que l'augmentation du pourcentage molaire du propionate s'accompagnait d'une diminution de la production de méthane ; ce qui concorde avec les résultats de Wolin (1960) et les relations stœchiométriques établies par Demeyer (1991).

Le monensin a provoqué in vitro, une diminution de la méthanogenèse, une augmentation du propionate alors que les autres AGV ont peu varié. En effet, le contenu du rumen, ayant été récolté $1 \mathrm{~h}$ après le repas et l'administration du monensin et la fermentation n'ayant duré que $3 \mathrm{~h}$, ce sont surtout les sucres facilement fermentescibles qui ont été fermentés. Des résultats similaires avaient été trouvés par Van Nevel et Demeyer (1977a) et Whetstone et al (1981). En accord avec Wolin (1960) et Demeyer (1991), la diminution de la méthanogenèse s'accompagne d'une augmentation de la production de propionate. Signalons que cette augmentation de la production de propionate due au monensin est plus importante chez le mouton $B$ que chez le mouton $A$ en période $P 30$ traduisant ainsi une différence de sensibilité au monensin des 2 moutons déjà observée in vivo.
La dose de $60 \mathrm{mg}$ de monensin a provoqué in vivo une augmentation significative du $\mathrm{pH}$ du milieu ruminal. Cette augmentation associée avec la diminution de la concentration en AGV totaux traduit une réduction de l'intensité des fermentations dans le rumen. Néanmoins, la dégradabilité in sacco de la matière sèche (MS) ne montre aucune action importante due au monensin. Cela pourrait faire penser à un effet sélectif exercé par le monensin sur la fermentation des monomères provenant de I'hydrolyse du substrat comme pour les protéines (Van Nevel et Demeyer, 1977a). Cette éventualité a été exclue dans un travail antérieur (Van Nevel et Demeyer, 1992). Par ailleurs, les sachets étant déposés dans le rumen $6 \mathrm{~h}$ après l'administration du monensin, il est possible que ce produit soit en grande partie métabolisé (Mary-Vauffrey et al, 1992) et/ou passé dans les autres compartiments du tube digestif, ce qui diminuerait son impact sur la dégradabilité in sacco du substrat.

L'arrêt de l'administration du monensin entraîne une chute momentanée de la dégradabilité in sacco de la matière sèche (MS) accompagnée d'un changement spectaculaire de tous les paramètres de la fermentation ayant fait l'objet de notre étude, signe d'une perturbation de la micropopulation du rumen.

L'ionophore n'a pas influencé la concentration en acide lactique dans le jus du rumen car celle-ci est négligeable. Ces résultats sont en accord avec ceux de Jouany et Sénaud (1978) qui révèlent que l'acide lactique est entièrement métabolisé dans le rumen $2 \mathrm{~h}$ après le début du repas.

La concentration en ammoniac dans le jus du rumen diminue avec l'administration du monensin, traduisant ainsi une diminution de la dégradation des protéines et des acides aminés dans le rumen comme constaté in vitro dans les incubations type «batch» (Adams et al, 1981 ; Fuller et Johnson, 1981 ; Russell et al, 1981 ; Van Nevel 
et Demeyer, 1977a) et «Rusitec» (Bogäert et al, 1990). La baisse de la concentration en ammoniac dans le jus du rumen se maintient tant qu'on administre encore du monensin.

Tous ces résultats de l'action du monensin aussi bien in vivo qu'in vitro nous permettent de conclure que dans nos conditions expérimentales il n'y a pas eu d'adaptation au monensin. Ces résultats semblent contradictoires avec ceux de Rumpler et al (1986) et Carmean et Johnson (1990). Néanmoins, soulignons qu'au cours de ces derniers travaux la production de méthane a été mesurée en chambre respiratoire sur des animaux nourris avec une ration plus riche en énergie que la nôtre et que les effets de cet ionophore sur les concentrations et les proportions des AGV n'ont pas été rapportés.

\section{REMERCIEMENTS}

Les auteurs souhaitent remercier S De Smet pour le traitement des résultats sur ordinateur, D Baeyens, A Maes, C Vermander et M Faquaet pour leur contribution dans la réalisation de ce travail, qui a été financé partiellement par le NFWO.

\section{RÉFÉRENCES}

Adams DC, Galyean ML, Kiesling HE, Wallace Joe D, Finkner MD (1981) Influence of viable yeast culture, sodium bicarbonate and monensin on liquid dilution rate, rumen fermentation and feedlot performance of growing steers and digestibility in lambs. $J$ Anim Sci 53, 780-789

Beede DK, Farlin SD (1977) Effects of antibiotics on apparent lactate and volatile fatty acid production: in vitro rumen fermentation studies. J Anim Sci 45, 385-392

Bogäert C, Jouany JP, Jeminet G (1990) Effects of the ionophore antibiotics monensin, monensin-propionate, abierixin and calcimycin on ruminal fermentations in vitro (Rusitec). Anim Feed Sci Technol 28 , 183-197

Burroughs WN, Frank A, Gerlaugh P, Bethke RM (1950) Preliminary observations upon factors influencing cellulose digestion by rumen microorganisms. $J$ Nutr $40,9-24$

Carmean BR, Johnson DE (1990) Persistence of monensin-induced changes in methane emissions and ruminal protozoa numbers in cattle. J Anim Sci 65 (suppl 1), 517

Chalupa W, Corbett W, Brethour JR (1980) Effects of monensin and amicloral on rumen fermentation. $\checkmark$ Anim Sci 51, 170-179

Crutzen PJ, Aselmann I, Seiler W (1986) Methane production by domestic animals, wild ruminants, and other herbivorous fauna and humans. Tellus 38B, 271-281

Demeyer D (1991) Quantitative aspects of microbial metabolism in the rumen and hundgut. In : Rumen Microbial Metabolism and Ruminant Digestion (JP Jouany, ed), INRA Éditions, Paris, 217-237

Demeyer DI, Van Nevel CJ (1979) Effect of defaunation on the metabolism of rumen microorganisms. Br J Nutr 42, 515-524

Dyer IA, Koes RM, Herlugson ML et al (1980) Effect of avoparcin and monensin on performance of beef cattle. J Anim Sci 48, 1138-1342

Fuller JR, Johnson DE (1981) Monensin and Lasalocid effects on fermentation in vitro. J Anim Sci53, 15741580

Haney ME, Hoehn MM (1967) Monensin, a new biological active compound. Antimicrob Agents Chemother, 349-352

Horn GW, Mader TL, Ambruster SL, Frahm RR (1981) Effect of monensin on ruminal fermentation, forage intake and weight gains of wheat pasture stocker cattle. J Anim Sci 52, 447-454

Jouany JP, Sénaud J (1978) Utilisation du monensin dans la ration des ruminants. II. Effets sur les fermentations et la population microbienne du rumen. Ann Zootech 27, 61-74

Joyner AE, Brown Jr LJ, Fogg TJ, Rossi RT (1979) Effect of monensin on growth, feed efficiency and energy metabolism of lambs. J Anim Sci 48, 1065-1069

Mary-Vaufrey F, Jouany JP, Dauphin G (1992) Biodegradation of monensin by rumen microorganisms in a Rusitec. Ann Zootech 41, 28

Poos MI, Hanson TL, Klopfenstein JJ (1979) Monensin effects on diet digestibility, ruminal bypass and microbial protein synthesis. J Anim Sci 53, 1516-1524

Raun AP, Cooley CO, Potter EL, Rathmacher RP, Richardson LF (1976) Effect of monensin on feed efficiency of feedlot cattle. J Anim Sci 43, 670-677

Richardson LF, Raun AP, Potter EL, Cooley CO, Rathmacher RP (1976) Effect of monensin on rumen fermentation in vitro and in vivo. J Anim Sci 43, 657-664

Rumpler WV, Johnson DE, Bates DB (1986) The effect of high dietary cation concentration on methanogenesis by steers fed diets with and without ionophores. J Anim Sci 62, 1737-1741 
Russell JB, Bottje WG, Cotta MA (1981) Degradation of protein by mixed cultures of rumen bacteria: identification of Streptococcus bovis as an actively proteolytic rumen bacterium. J Anim Sci53, 242-252

Sakauch R, Wakita M, Hoshino S (1979) Effect of monensin on ruminal VFA and ammonia levels and microbes of finishing cattle. Jpn J Zootech Sci 50, 369-374

Shibata M, Terada F, Iwasaki K, Kurihara M, Nishida T (1992) Methane production in heifers, sheeps and goats consuming diets of various hay-concentrate ratios. Anim Sci Technol (Jpn) 63, 1221-1227

Stanier G, Davies A (1981) Effects of the antibiotic monensin and an inhibitor of methanogenesis on in vitro continuous rumen fermentations. Br J Nutr 45, 567-578

Thornton JH, Owens FN (1981) Monensin supplementation and in vivo methane production by steers. $J$ Anim Sci 52, 628-634

Todd GC, Novilla MN, Howard LC (1984) Comparative toxicology of monensin sodium in laboratory animals. J Anim Sci 58, 1512-1517
Van Nevel CJ, Demeyer DI, Henderickx HK, Martin JA (1970) A simple method for the simultaneous determination of gas production and volatile fatty acid concentration in the rumen. Zeitschr Tierphys Tierernähr Futtermittelk 26, 91-100

Van Nevel CJ, Demeyer DI (1977a) Effect of monensin on rumen fermentation in vitro. Appl Env Microbio/34, 251-257

Van Nevel CJ, Demeyer DI (1977b) Determination of rumen microbial growth in vitro from ${ }^{32} \mathrm{P}$ labelled phosphate incorporation. Br J Nutr 38, 101-114

Van Nevel CJ, Demeyer DI (1992) Influence of antibiotics and a deaminase inhibitor on volatile fatty acids and methane production from detergent washed hay and soluble starch by rumen microbes in vitro. Anim Feed Sci Technol 37, 21-31

Whetstone HD, Davis CL, Bryant MP (1981) Effect of monensin on breakdown of protein by ruminal microorganisms in vitro. J Anim Sci 53, 803-809

Wolin MJ (1960) A theoretical rumen fermentation balance. J Dairy Sci 43, 1452-1459 